\title{
Acidic drainage in carbonate rocks
}

\author{
Boris Ryzhenko ${ }^{1, *}$, Oksana Limanseva ${ }^{1}$, and Elena Cherkasova $^{1}$ \\ ${ }^{1}$ Vernadsky Institute of Geochemistry and Analytical Chemistry Russian Academy of Sciences, \\ Moscow
}

\begin{abstract}
Thermodynamic simulation of the system "Rocks of Pavlovskoe polymetallic deposit - water - atmosphere gases" predicts aqueous concentrations from mineral solubilities and speciation with the HCh geochemical code. It is shown the model solutions are alkaline and rich in some microelements (As, $\mathrm{Cd}, \mathrm{Se}, \mathrm{Co}, \mathrm{Cr}, \mathrm{Cu}, \mathrm{Ni}, \mathrm{Pb}, \mathrm{Ga}, \mathrm{Ge}, \mathrm{Sr}, \mathrm{In}$ ); their concentrations are overcome MPC, especially on the ore storage site.
\end{abstract}

Currently, the environment degradation due to mining can be predicted using hydrogeochemistry background. It was shown by S.R. Kraynov et al. [1, 2] the natural water chemical composition in the Earth's crust is determined by chemical and mineralogical composition of the rocks, their reaction with water, rock to water ratios $(\mathrm{R} / \mathrm{W})$, thermodynamic closeness/openness of the system with respect to $\mathrm{CO}_{2}$ and $\mathrm{O}_{2}$ and the system temperature and pressure.

Petrochemical type of rock is responsible for the aqueous phase composition. The rock to water ratio depends on rock porosity, groundwater flow velocity and, generally, water exchange. The next step in geochemical simulation will be equilibrium-kinetic modeling developed by Mironenko and Zolotov [3].

It was shown previously $[4,5]$ that silicic rocks, formed by minerals with a low rate of dissolution, are the most favorable for producing acidic drainage especially from finely crushed rocks which are exposed to intermittent water flow. Water films with long residence times are favorable for enrichment by sulfide oxidation products in fine grained rocks. Active water flow is favorable for washing out oxidation products and forming acid drainage. The drainage solutions are enriched in microelements during low flow periods and at the higher $\mathrm{R} / \mathrm{W}$ ratio. In this publication we want to compare the drainage solution compositions in silicate and in carbonate rocks. The rate of carbonate dissolution in water is faster than that of silicates. So it is easy to predict complete neutralization of sulfuric acid formed by carbonates.

\footnotetext{
*Corresponding author: ryzhenko@geokhi.ru
} 


\section{Geochemical conditions needed for simulation}

On the Pavlovskoe deposit site all geological systems from Cambrian to Permian are present. They are marine, volcanic and volcano-sedimentary. Igneous rocks are basic composition mostly [6, 7].

Lead-zinc ore fields are located in a river basin containing mostly carbonate and terrigenous-carbonate rocks. The ore field belongs to mirgalimsay type (lead-zinc stratiform in carbonates). The Gribovska suite of $250-300 \mathrm{~m}$ thickness is composed of organogenic limestones and dolomites. There are three ore blocks. Ores are divided into several mineral types: pyrite, pyrite-galena-sphalerite and, the largest one, galena-sphlerite-pyrite [8,9].

Common impurities in sphalerites are the following $\mathrm{Cd}, \mathrm{Ag}, \mathrm{Pb}, \mathrm{Ga}$, also $\mathrm{Co}, \mathrm{Ge}, \mathrm{Sb}$, $\mathrm{Sn}, \mathrm{Sb}, \mathrm{Ge}, \mathrm{Co}, \mathrm{Ag}, \mathrm{Bi}$. The $\mathrm{Cd}$ content varies from 0 to $0.22 \%$ ( average $0.1 \%$ ) and $\mathrm{Bi}$ content is up to $0.16 \%$.

Lead in ore matrix is up to $20 \%$; typical impurities are $\mathrm{Ag}, \mathrm{Sb}, \mathrm{Cd}, \mathrm{Co}, \mathrm{Zn}, \mathrm{Bi}$ and $\mathrm{Sn}$; $\mathrm{Ag}$ and $\mathrm{Cd}, \mathrm{Sb}$, partly $\mathrm{Ag}$ and $\mathrm{Bi}$ and $\mathrm{Zn}$, partly $\mathrm{Cd}$; $\mathrm{Sn}$ is due to mineral inclusions in galena and sphalerite. Carbonates and quartz are among the main minerals, and albite, schist silicates, and apatite are less common. Calcites are from sedimentary rocks, it has impurities of $\mathrm{Mg}, \mathrm{Mn}, \mathrm{Fe}, \mathrm{Zn}$. In oxidised ores iron-containing minerals are predominant. So in groundwaters 14 elements are possible, some are of high toxicity; they are $\mathrm{Cd}, \mathrm{Pb}$, $\mathrm{Co}, \mathrm{Sb}, \mathrm{Al}, \mathrm{Si}, \mathrm{Na}, \mathrm{Fe}, \mathrm{Mn}, \mathrm{Ag}, \mathrm{Zn}, \mathrm{Ga}, \mathrm{Ge}, \mathrm{Sn}, \mathrm{Ni}$, In, Bi, Sn, P, also sulfates, silicates etc. Mineralization of water on Pavlovskoe deposit field is from 0.2 to $1.0 \mathrm{~g} / \mathrm{l}$; water type is $\mathrm{HCO}_{3}-\mathrm{SO}_{4}-\mathrm{Ca}-\mathrm{Mg}$; $\mathrm{pH}$ is neutral or a little bit alkaline. There is no correlation between aqueous concentrations of $\mathrm{Li}, \mathrm{Na}, \mathrm{Mg}, \mathrm{Al}, \mathrm{P}_{\text {total }}, \mathrm{S}_{\text {total }}, \mathrm{K}, \mathrm{Ca}, \mathrm{Cr}, \mathrm{Mn}, \mathrm{Fe}, \mathrm{Co}, \mathrm{Ni}$ and their bottom sediment concentrations.

\section{Acidic drainage on Pavlovskoe deposit}

Using HCh Code [10] (Y. Shvarov, Geochemistry Department of Moscow State University), the equilibrium state of the system "rocks of Pavlovskoe deposit - water atmosphere gases" was calculated $(\mathrm{pH}, \mathrm{Eh}$, aqueous species concentrations and mineral assemblages).

Acidic solution formation occurs in accordance with research [11]

$2 \mathrm{FeS}_{2}$ (pyrite) $+7.5 \mathrm{O}_{2}(\mathrm{~g})+5 \mathrm{H}_{2} \mathrm{O}=2 \mathrm{FeOOH}(\mathrm{s})+4 \mathrm{H}_{2} \mathrm{SO}_{4}(\mathrm{aq})$,

with the rate $\approx 5 \times 10^{-10}$ mole. $\mathrm{cm}^{-2} \mathrm{c}^{-1}$ (at $25^{\circ} \mathrm{C}$ ). Bacteria quicken oxidation. There are several scenarios of the oxidation process

(1) Sulfuric acid is neutralized by rock minerals. During low flow, $\mathrm{pH}$ is higher than neutral value. The acidic solution is neutralized by carbonate rocks (fig. 1). The elements of dissolved minerals increase in their concentrations with increasing R/W ratio.

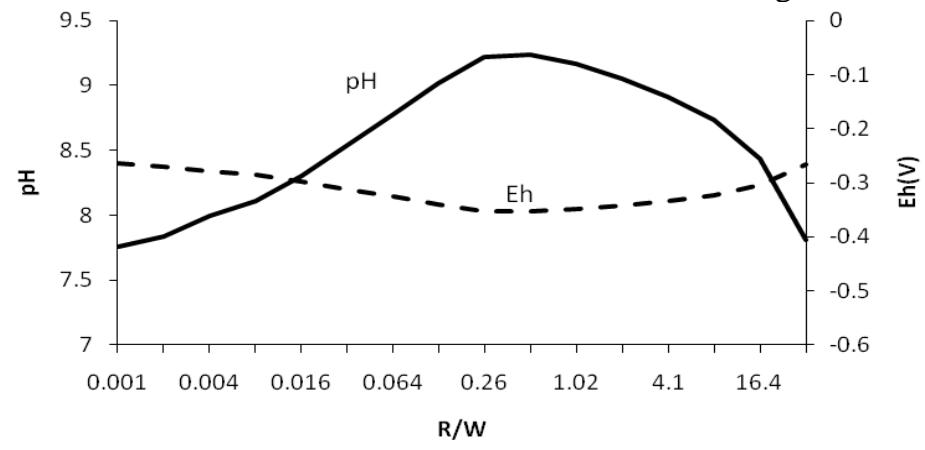

Fig. 1. Eh-pH of drainage waters versus $\mathrm{R} / \mathrm{W}$ at $\mathrm{P} \mathrm{O}_{2} \geq 10^{-70}$ bar and $\mathrm{P} \mathrm{CO}_{2}=10^{-3}$ bar. 
(2) Acid is neutralized by rock more slowly. The $\mathrm{pH}$ begins acidic. The drainage solution $\mathrm{pH}$ depends on contact time of rock and aqueous solution and $\mathrm{R} / \mathrm{W}$ (fig. 2). After 3 years the drainage has become neutral.

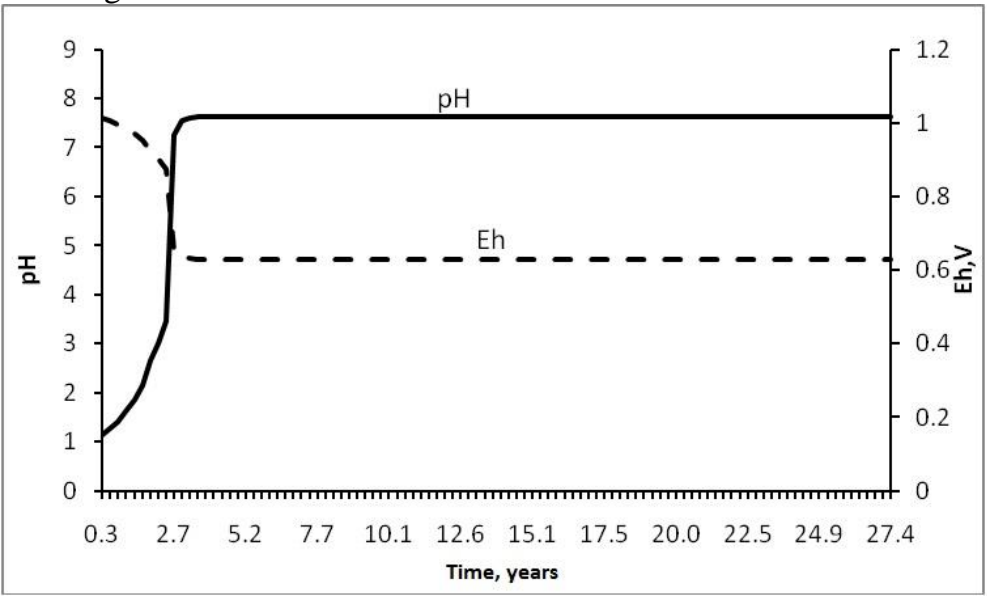

Fig. 2. Eh-pH of drainage waters as interaction time of water and water-bearing rock at $\mathrm{R} / \mathrm{W}=0.0003$, $\mathrm{P} \mathrm{O}_{2}=10^{-1}$ bar and $\mathrm{P} \mathrm{CO}_{2}=10^{-3}$ bar).

\section{Thermodynamic simulation of the system "Rocks of Pavlovskoe deposit-water-atmospheric gases"}

Information used for simulation: a) temperature $10^{\circ} \mathrm{C}$ and atmosphere pressure; b) The system is open to atmosphere $\mathrm{O}_{2}$ and $\mathrm{CO}_{2}$, c) pyrite-galena-sphalerite ore association, d) mass ratio of rock and water $\log (\mathrm{R} / \mathrm{W}$ ) changes from -4 to +0.8 (range of possible water residence times for various sites of deposit). The results are in table 1 . The aqueous solution composition are controlled by the solubility of ankerite, barite, calcite, cerussite, chalcocite, dolomite, fluorite, galena, hausmannite, goethite, gypsum, microcline, quartz, mercury, rhodochrosite, smithsonite, sphalerite, stilbite, strontianite, witherite, greenockite.

Table 1. Concentrations of dissolved chemical elements and mineral masses resulted from oxidative dissolution pyrite of Pavlovskoe polymetallic deposit.

\begin{tabular}{|c|c|c|c|c|c|c|}
\hline $\mathrm{R} / \mathrm{W}$ & 0.0001 & 0.001 & 0.01 & 0.1 & 1 & 6.3 \\
\hline $\mathrm{pH}$ & 7.899 & 7.812 & 7.546 & 7.542 & 7.519 & 7.463 \\
\hline $\mathrm{Eh}(\mathrm{V})$ & -0.185 & 0.002 & 0.186 & 0.326 & 0.496 & 0.683 \\
\hline \multicolumn{7}{|c|}{ Aqueous solution, total mg/l } \\
\hline $\mathrm{Al}$ & $2.23 \mathrm{E}-06$ & $2.98 \mathrm{E}-07$ & $1.88 \mathrm{E}-07$ & $1.90 \mathrm{E}-07$ & $1.97 \mathrm{E}-07$ & $1.92 \mathrm{E}-07$ \\
\hline $\mathrm{As}$ & $5.82 \mathrm{E}-05$ & $6.23 \mathrm{E}-04$ & $\mathbf{5 . 5 5 E}-03$ & $\mathbf{3 . 4 5 E}-02$ & $\mathbf{3 . 1 7 E}-01$ & $\mathbf{5 . 1 6 E}+00$ \\
\hline $\mathrm{Ba}$ & $6.21 \mathrm{E}-04$ & $1.33 \mathrm{E}-03$ & $4.28 \mathrm{E}-03$ & $4.52 \mathrm{E}-03$ & $6.12 \mathrm{E}-03$ & $1.27 \mathrm{E}-02$ \\
\hline $\mathrm{Ca}$ & $3.52 \mathrm{E}+01$ & $1.00 \mathrm{E}+02$ & $5.84 \mathrm{E}+02$ & $6.19 \mathrm{E}+02$ & $8.51 \mathrm{E}+02$ & $2.07 \mathrm{E}+03$ \\
\hline $\mathrm{Cd}$ & $8.66 \mathrm{E}-08$ & $1.18 \mathrm{E}-05$ & $1.05 \mathrm{E}-04$ & $6.51 \mathrm{E}-04$ & $\mathbf{5 . 9 9 E}-03$ & $\mathbf{9 . 7 4 E - 0 2}$ \\
\hline $\mathrm{Co}$ & $1.10 \mathrm{E}-04$ & $1.18 \mathrm{E}-03$ & $1.05 \mathrm{E}-02$ & $6.51 \mathrm{E}-02$ & $\mathbf{5 . 9 9 E}-01$ & $\mathbf{9 . 7 4 E + 0 0}$ \\
\hline $\mathrm{Cr}$ & $1.54 \mathrm{E}-03$ & $1.65 \mathrm{E}-02$ & $1.47 \mathrm{E}-01$ & $\mathbf{9 . 1 2 E}-01$ & $\mathbf{8 . 3 8 E}+\mathbf{0 0}$ & $\mathbf{1 . 3 6 E + 0 2}$ \\
\hline $\mathrm{Cu}$ & $1.56 \mathrm{E}-10$ & $4.70 \mathrm{E}-06$ & $4.19 \mathrm{E}-05$ & $2.60 \mathrm{E}-04$ & $2.39 \mathrm{E}-03$ & $3.90 \mathrm{E}-02$ \\
\hline $\mathrm{Fe}$ & $1.12 \mathrm{E}-02$ & $4.27 \mathrm{E}-05$ & $2.50 \mathrm{E}-07$ & $1.74 \mathrm{E}-08$ & $1.72 \mathrm{E}-08$ & $1.96 \mathrm{E}-08$ \\
\hline $\mathrm{Ga}$ & $3.46 \mathrm{E}-05$ & $3.71 \mathrm{E}-04$ & $3.30 \mathrm{E}-03$ & $2.05 \mathrm{E}-02$ & $1.89 \mathrm{E}-01$ & $3.07 \mathrm{E}+00$ \\
\hline
\end{tabular}




\begin{tabular}{|c|c|c|c|c|c|c|}
\hline $\mathrm{Ge}$ & $9.10 \mathrm{E}-05$ & $9.73 \mathrm{E}-04$ & $8.68 \mathrm{E}-03$ & 5.39E-02 & $4.96 \mathrm{E}-01$ & $8.06 \mathrm{E}+00$ \\
\hline $\mathrm{Hg}$ & $1.14 \mathrm{E}-22$ & $1.23 \mathrm{E}-16$ & $1.92 \mathrm{E}-09$ & $5.86 \mathrm{E}-07$ & $5.39 \mathrm{E}-06$ & $8.77 \mathrm{E}-05$ \\
\hline $\mathrm{K}$ & $6.99 \mathrm{E}-02$ & $7.48 \mathrm{E}-01$ & $6.67 \mathrm{E}+00$ & $4.14 \mathrm{E}+01$ & $3.81 \mathrm{E}+02$ & $6.06 \mathrm{E}+03$ \\
\hline $\mathrm{Mg}$ & $5.48 \mathrm{E}+00$ & $3.21 \mathrm{E}+01$ & $1.83 E+02$ & $1.94 \mathrm{E}+02$ & $2.74 \mathrm{E}+02$ & $1.27 \mathrm{E}+03$ \\
\hline $\mathrm{Na}$ & $4.58 \mathrm{E}-03$ & $4.90 \mathrm{E}-02$ & 4.37E-01 & $2.71 \mathrm{E}+00$ & $6.41 \mathrm{E}+00$ & $1.64 \mathrm{E}-01$ \\
\hline $\mathrm{Ni}$ & $6.59 \mathrm{E}-04$ & $7.05 \mathrm{E}-03$ & 6.29E-02 & 3.91E-01 & $3.59 \mathrm{E}+00$ & $5.84 \mathrm{E}+01$ \\
\hline $\mathrm{Pb}$ & $1.67 \mathrm{E}-04$ & 4.15E-01 & 7.19E-01 & 7.39E-01 & 8.98E-01 & $1.42 \mathrm{E}+01$ \\
\hline $\mathrm{SO}_{4}$ & $2.54 \mathrm{E}+01$ & $2.65 \mathrm{E}+02$ & $1.54 \mathrm{E}+03$ & $1.63 E+03$ & $2.14 \mathrm{E}+03$ & $4.16 \mathrm{E}+03$ \\
\hline $\mathrm{Se}$ & $1.32 \mathrm{E}-05$ & $1.42 \mathrm{E}-04$ & $1.26 \mathrm{E}-03$ & $7.84 \mathrm{E}-03$ & $7.21 \mathrm{E}-02$ & $1.17 \mathrm{E}+00$ \\
\hline $\mathrm{Si}$ & $8.11 \mathrm{E}-01$ & $1.31 \mathrm{E}+00$ & $1.31 \mathrm{E}+00$ & $1.31 \mathrm{E}+00$ & $1.31 \mathrm{E}+00$ & $1.41 \mathrm{E}+00$ \\
\hline $\mathrm{Sr}$ & $9.89 \mathrm{E}-04$ & $1.06 \mathrm{E}-02$ & $9.43 \mathrm{E}-02$ & $5.86 \mathrm{E}-01$ & $5.39 \mathrm{E}+00$ & $2.93 E+01$ \\
\hline $\mathrm{Te}$ & $1.82 \mathrm{E}-06$ & $1.95 \mathrm{E}-05$ & $1.74 \mathrm{E}-04$ & $1.08 \mathrm{E}-03$ & $9.94 \mathrm{E}-03$ & 1.62E-01 \\
\hline $\mathrm{Zn}$ & $6.11 \mathrm{E}-03$ & $9.96 \mathrm{E}+00$ & $5.49 \mathrm{E}+01$ & $5.77 \mathrm{E}+01$ & $7.65 \mathrm{E}+01$ & $3.21 \mathrm{E}+02$ \\
\hline \multicolumn{7}{|c|}{ Mineral assemblage, moles } \\
\hline Ankerite & 4.39E-05 & 0 & 0 & 0 & 0 & 0 \\
\hline Barite & 0 & 0 & $6.84 \mathrm{E}-04$ & $4.23 \mathrm{E}-03$ & $3.77 \mathrm{E}-02$ & $4.04 \mathrm{E}-01$ \\
\hline Calcite & 0 & $6.27 \mathrm{E}-03$ & $5.35 \mathrm{E}-02$ & $2.52 \mathrm{E}-01$ & $2.13 \mathrm{E}+00$ & $2.26 \mathrm{E}+01$ \\
\hline Cerussite & 0 & $1.32 \mathrm{E}-05$ & $1.32 \mathrm{E}-04$ & 8.37E-04 & 7.49E-03 & $8.02 \mathrm{E}-02$ \\
\hline Chalcocite & $3.46 \mathrm{E}-12$ & 0 & 0 & 0 & 0 & 0 \\
\hline Dolomite & 0 & $1.09 \mathrm{E}-03$ & $1.40 \mathrm{E}-02$ & $1.25 \mathrm{E}-01$ & $1.18 \mathrm{E}+00$ & $1.27 \mathrm{E}+01$ \\
\hline Fluorite & 0 & 0 & 0 & $4.41 \mathrm{E}-05$ & $5.56 \mathrm{E}-04$ & $6.17 \mathrm{E}-03$ \\
\hline Galena & $1.42 \mathrm{E}-06$ & 0 & 0 & 0 & 0 & 0 \\
\hline Hausmannite & 0 & 0 & 0 & 0 & 0 & $3.76 \mathrm{E}-03$ \\
\hline Goethite & 0 & $4.72 \mathrm{E}-04$ & $4.21 \mathrm{E}-03$ & $2.61 \mathrm{E}-02$ & $2.32 \mathrm{E}-01$ & $2.49 \mathrm{E}+00$ \\
\hline Gypsum & 0 & 0 & $5.84 \mathrm{E}-03$ & $1.51 \mathrm{E}-01$ & $1.53 \mathrm{E}+00$ & $1.66 \mathrm{E}+01$ \\
\hline Microcline & 0 & 0 & 0 & 0 & 0 & $2.25 \mathrm{E}-03$ \\
\hline Quartz & 0 & $2.62 \mathrm{E}-04$ & $2.71 \mathrm{E}-03$ & 1.70E-02 & $1.52 \mathrm{E}-01$ & $1.63 \mathrm{E}+00$ \\
\hline Mercury & $4.93 \mathrm{E}-15$ & $5.28 \mathrm{E}-14$ & $4.57 \mathrm{E}-13$ & 0 & 0 & 0 \\
\hline Rhodochrosite & 0 & 0 & 0 & 0 & $7.83 \mathrm{E}-04$ & 0 \\
\hline Smithsonite & 0 & $2.60 \mathrm{E}-05$ & $7.49 \mathrm{E}-04$ & $8.96 \mathrm{E}-03$ & $8.66 \mathrm{E}-02$ & $9.36 \mathrm{E}-01$ \\
\hline Sphalerite & $1.66 \mathrm{E}-05$ & 0 & 0 & 0 & 0 & 0 \\
\hline Stilbite & $1.99 \mathrm{E}-08$ & $2.13 \mathrm{E}-07$ & $1.90 \mathrm{E}-06$ & $1.18 \mathrm{E}-05$ & $1.05 \mathrm{E}-04$ & 0 \\
\hline Strontianite & 0 & 0 & 0 & 0 & 0 & $4.24 \mathrm{E}-04$ \\
\hline Witherite & $7.16 \mathrm{E}-06$ & $7.67 \mathrm{E}-05$ & 0 & 0 & 0 & 0 \\
\hline Greenockite & $9.00 \mathrm{E}-12$ & 0 & 0 & 0 & 0 & 0 \\
\hline
\end{tabular}

As it is seen in table 1 the model element concentrations increase with R/W ratio up to the MPC in potable waters. 


\section{Conclusion}

The results show that danger of ore deposit drainage waters is not its high acidity but high concentrations of microelements (As, $\mathrm{Cd}, \mathrm{Se}, \mathrm{Co}, \mathrm{Cr}, \mathrm{Cu}, \mathrm{Ni}, \mathrm{Pb}, \mathrm{Ga}, \mathrm{Ge}, \mathrm{Sr}$ ), especially on ore storage [or waste storage] sites.

Thermodynamic simulation of the system "rocks of Pavlovskoe ore deposit-wateratmosphere gases" shows:

(1) There is increase of aqueous concentrations of $\mathrm{Cr}, \mathrm{Sr}, \mathrm{Ni}, \mathrm{Co}, \mathrm{Ge}, \mathrm{As}, \mathrm{Ga}, \mathrm{Se}, \mathrm{Te}$, $\mathrm{Cd}, \mathrm{Cu}$ as water-flow rate decreases ( $\mathrm{R} / \mathrm{W}$ simulation parameter increases).

(2) The element aqueous concentrations are the same as their values in the deposit surface waters. So simulated element concentrations may be used for prognosis of environment pollution at mining.

(3) The drainage solution has near neutral $\mathrm{pH}$ but it is enriched substantially in microelements ( $\mathrm{Zn}, \mathrm{Pb}, \mathrm{Sr}, \mathrm{Ni}, \mathrm{As}, \mathrm{Cr}, \mathrm{Co}, \mathrm{Se}, \mathrm{Te}, \mathrm{Cd})$.

\section{References}

1. S.R. Kraynov, B.N. Ryzhenko, V.M. Shvets, Geochemistry of ground waters (CentrLitNefteGas, 2012)

2. Geological evolution and selforganization of water-rock system (SB of RAN, 2007. 2)

3. M.V. Mironenko, M.Y. Zolotov, Geochemistry International. 50. 1, 1-7 (2012)

4. B.N. Ryzhenko, T.P. Belova, A.E. Ryabenko, E.V. Cherkasova, Water Resources. 43. 7, 964-973 (2016)

5. O.A. Limantseva, B.N. Ryzhenko, E.V. Cherkasova, Geochemistry International. 53. 10, 922-936 (2015)

6. Severnaya Zemla. Geological Structure and Mineragenesis (Editor I.S.Granberg, V.I Ushakov St. Petersburg VNII Okeanologiya, 2000)

7. Geology and ores mineral resources of Russia (Editors I.S. Granberg, I.A. Ivanova, U.E. Pogrebetsky V.5, Book 1. Arctic seas. St. Petersburg. VSEGEI, 2000)

8. T.A. Gubina (Avtoreferat of PhD Dissertation of geology-mineralogy science, St. Petersburg, 2000)

9. V.O. Ilchenko (Dissertation on geological and mineralogical science: 25.00.05: St. Peterburg. State Public Library Number 61:05-4/58, 2004)

10. Yu.V. Shvarov. Geochemistry International. 46. 8, 834-839 (2008)

11. M.A. Williamson, J.D. Rimstidt, Geochem. Cosmochem. Acta. 58. 2, 5443-5454 (1994) 